\title{
Correction to: Visual Privacy Management
}

\author{
Mattia Salnitri (D), Jan Jürjens (D), Haralambos Mouratidis (D), \\ Loredana Mancini (D), and Paolo Giorgini (D)
}

\section{Correction to:}

M. Salnitri et al. (Eds.): Visual Privacy Management, LNCS 12030, https://doi.org/10.1007/978-3-030-59944-7

In the original version of this book, the name of the second editor was not correct. This has now been rectified. 